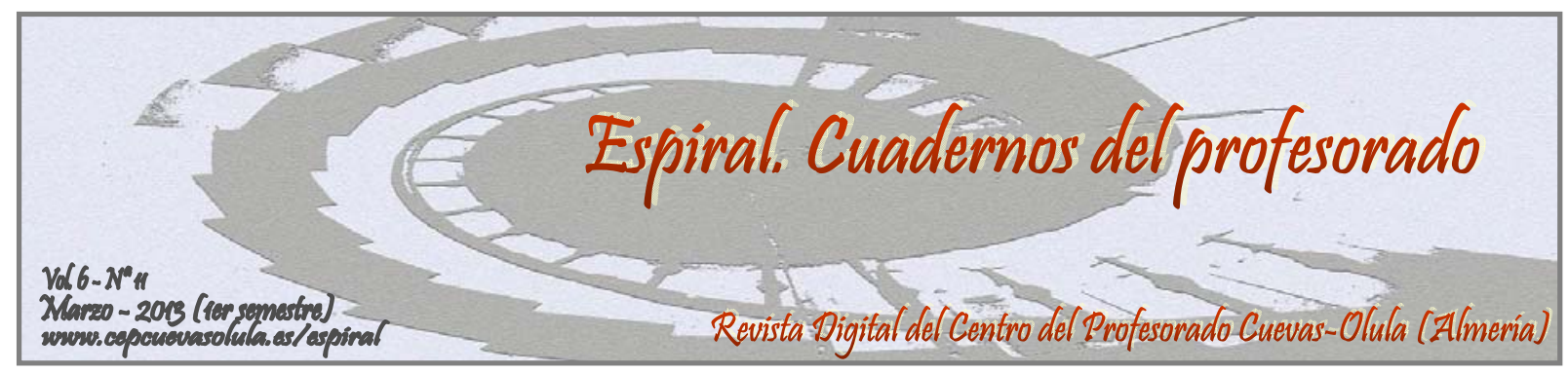

\title{
EL ATLETISMO EN EL SISTEMA EDUCATIVO
}

\author{
ATHLETICS IN EDUCATION SYSTEM
}

\section{Alfonso Valero Valenzuela y Alberto Gómez Mármol}

Facultad de Ciencias del Deporte, Universidad de Murcia, España

RESUMEN: En este trabajo se analizan las posibilidades educativas que el atletismo puede aportar a los jóvenes escolarizados, trascendiendo de la orientación hacia la competición que busca obtener un máximo rendimiento. Así, el atletismo, dentro del marco del sistema educativo debe ser considerado como un medio que conduzca hacia la iniciación deportiva con un carácter formativo. Estas posibilidades educativas se confrontan con la legislación actual a nivel nacional y, de forma más específica, con la normativa vigente en la Región de Murcia. De este modo, se plantea una secuenciación de las disciplinas que componen el atletismo desde el $3^{\text {er }}$ ciclo de primaria y a lo largo de la etapa secundaria y, a partir de una hipotética unidad didáctica aplicada en $1^{\circ}$ de Educación Secundaria Obligatoria, la relación del atletismo con las enseñanzas mínimas. Además se presenta su vínculo con los objetivos de materia y etapa, con las competencias básicas, con los contenidos y, finalmente, con los criterios de evaluación. Por tanto, se aporta una propuesta para la implementación de la iniciación al atletismo en cualquier centro educativo.

Palabras clave: Educación secundaria, iniciación deportiva, legislación educativa, Educación Física

ABSTRACT: In this work, the educational capabilities that athletics can provide to scholar youth are analysed, going beyond the competition orientation which looks forward the best performance. Thus, athletics must be considered as a vehicle that leads toward an educational sport initiation. These educational capabilities are faced to the current national laws and, more specifically, to the current Region of Murcia laws. In this way, athletics' disciplines sequence from $3^{\text {rd }}$ stage of primary education and through secondary education is presented and, starting from an hypothetical teaching unit for the $1^{\text {st }}$ grade of secondary education, the relationship between athletics and minimum teachings is also presented. Furthermore its link with subject and stage scholar aims, with basics skills, with contents and, finally, with evaluation criterions is showen. Thus, a proposal for the implementation of athletics initiation in any scholar centre is provided.

Key words: Secondary education, sport initiation, educational law, physical education

Valero Valenzuela, A., y Gómez Mármol, A. (2013). El atletismo en el sistema educativo. Espiral. Cuadernos del Profesorado, 6(11), 46-58. Disponible en: http://www.cepcuevasolula.es/espiral

Fecha de recepción: 31/10/2012

Fecha de aceptación: 26/02/2013
Enviar correspondencia a: alberto.gomez1@um.es 


\section{1.- INTRODUCCIÓN}

Tradicionalmente el enfoque que se ha adoptado del atletismo en las clases de Educación Física se ha basado en la utilización de los sistemas de entrenamiento propios del atletismo de competición, sistemas que se basan en la especialización y en la automatización, requiriendo grandes cantidades de práctica regular, cuyo fin último es la obtención del máximo rendimiento o la mayor eficacia posible.

La iniciación al atletismo dentro del sistema educativo sólo se debe enfocar desde el punto de vista puramente educativo, en el que el atletismo actúa como mediador para la consecución del objetivo de la formación integral del alumnado, siendo los juegos y la técnica deportiva los elementos empleados para su alcance. Siguiendo al Real Decreto 1631/2006, se especifica el enfoque que ha de tener la Educación Física; concretamente detalla "tiene un carácter integrador e incluye una multiplicidad de funciones: cognitivas, expresivas, comunicativas y de bienestar" (p. 710), y más adelante haciendo referencia al bloque de "Juegos y deportes", concreta que "tiene sentido en la Educación Física por su carácter educativo, por ser un instrumento adecuado para la mejora de las capacidades que pretende esta materia y porque supone actividades que fomentan la colaboración, la participación y el desarrollo de actitudes dirigidas hacia la solidaridad, la cooperación y la no discriminación". Queda claro que el eje del proceso de enseñanza - aprendizaje es el alumnado y el atletismo un medio adecuado para favorecer la adquisición de una serie de competencias básicas y la consecución de los objetivos generales de etapa.

Tanto en el Real Decreto 1631/2006, de 29 de diciembre, por el que se establecen las enseñanzas mínimas correspondientes a la Educación Secundaria Obligatoria (ESO), como en el Decreto 291/2007, de 14 de septiembre, por el que se establece el currículo de la Educación Secundaria Obligatoria en la Comunidad Autónoma de la Región de Murcia, se contemplan objetivos, contenidos y criterios de evaluación relacionados con el conocimiento y la realización de actividades deportivas individuales, así como la evaluación y mejora de las capacidades específicas de un deporte individual. Especialmente en el Decreto 291/2007 de la Región de Murcia, dentro del bloque de "Juegos y deportes. Cualidades motrices personales", aparece el contenido durante los cursos de $1^{\circ}$ y $2^{\circ}$ "Práctica de juegos regulados y adaptados, convencionales y modificados, populares y tradicionales, que faciliten el aprendizaje de los fundamentos técnicos/tácticos y las estrategias de ataque y defensa comunes a los deportes individuales, de adversario y colectivos” p. 27211-27212), quedando latente la intencionalidad desde el sistema educativo para que durante el primer ciclo de la ESO, se realice una iniciación deportiva a través de los juegos. Se busca una transición desde el juego de forma genérica al juego deportivo y a la iniciación deportiva. Esto conduce a la idea de que el proceso de iniciación al atletismo tiene cabida y es conveniente incorporarlo en el currículum de la enseñanza de la ESO, dando una mayor amplitud a los contenidos propios de los “Juegos y Deportes”, haciendo que el alumnado conozca la verdadera raíz social que el atletismo encierra y las conductas humanas que a través del mismo se pueden adquirir.

En cursos posteriores ( $3^{\circ}$ y $4^{\circ}$ de ESO), el bloque de "Juegos y Deportes. Cualidades motrices personales" alberga contenidos más concretos para los deportes como en el caso de $3^{\circ}$, la "Práctica de actividades encaminadas al aprendizaje de un deporte individual, de adversario o colectivo" (pg. 27213), que buscan la iniciación deportiva en una disciplina concreta, persiguiendo una mayor profundización en el deporte seleccionado. Para $4^{\circ}$ de ESO, se concreta aún más y se habla de perfeccionamiento, citando textualmente "realización de actividades encaminadas al aprendizaje y al perfeccionamiento del deporte individual, de adversario o colectivo" (pg. 27214), lo que permite al docente ahondar en un deporte seleccionado, si bien, los criterios de evaluación para este tipo de contenidos será "participar en la organización y puesta en práctica de torneos en los que se practicarán deportes y actividades físicas realizadas a lo largo de la etapa” (p. 27215). Por tanto, poco a poco, la iniciación deportiva basada en el juego se va convirtiendo en una mejora de habilidades técnico-tácticas más específicas, para ponerlas en práctica en un ambiente lúdico-festivo como serían los torneos, en los que el alumnado colabora tanto en la organización de situaciones deportivas competitivas de los diferentes deportes como mediante su participación activa en las mismas. 
Por tanto, el objetivo de este trabajo consiste en analizar, minuciosamente y mediante ejemplos, las posibilidades educativas con las que el atletismo puede contribuir a la consecución de una formación integral de los alumnos; entendida la formación integral en los términos que establece la legislación educativa vigente.

\section{2.- SITUACIÓN ACTUAL DEL ATLETISMO Y SU METODOLOGÍA DE ENSEÑANZA EN LA INICIACIÓN DEPORTIVA}

Los esfuerzos realizados por especialistas en el campo de la enseñanza del atletismo no están siendo correspondidos en la práctica con una adecuado grado de motivación, con el deseado compromiso activo, ni con el nivel de ejecución óptimo durante las prácticas. Tras un análisis de la problemática desde el punto de vista didáctico, como causas que determinan la falta de interés que el alumnado siente por la práctica del atletismo destacan la metodología desfasada, la rígida estructura de las sesiones y una motivación demasiado centrada en elementos externos (Valero, 2004).

Frente a la metodología tradicional recogida en gran cantidad de libros y manuales básicos de atletismo (Álvarez, 1994; Calzada, 1999; Hegedus, 1988; Hubiche y Pradet, 1999; Murer, 1992; Polischuk, 1996; Rius, 1989; Schmolinsky, 1981; Vinuesa y Coll, 1997), surgen nuevas propuestas metodológicas, entre las que destaca la enseñanza comprensiva del deporte, donde adquiere gran relevancia la conciencia táctica y la toma de decisiones (Méndez, 2009).

Otros planteamientos en esta misma línea es la denominada Educación Deportiva. Se trata de un modelo de enseñanza que surge con el propósito de estimular experiencias de práctica deportiva auténticas (Siedentop, 1994, p. 4; Siedentop, Hastie y Van der Mars, 2011, p. 6). Experiencias en las que los jóvenes practicantes vivencien el deporte desde una perspectiva global, que desarrolle su competencia motriz, su cultura deportiva y su entusiasmo por la práctica. Hasta el momento, todas las revisiones realizadas no se han centrado en un deporte concreto (Curtner-Smith y Sofo, 2004; Hastie, Martínez de Ojeda y Calderón, 2011; Kinchin, 2006) sino que abordan los trabajos que han aplicado el Modelo de Educación Deportiva en diferentes contextos (fundamente escolar) y con distintos contenidos (desde el rugby y el voleibol hasta la gimnasia artística y el atletismo).

Por otro lado, desde la Real Federación Española de Atletismo (RFEA), se ha elaborado la guía denominada "Jugando al Atletismo", desarrollada por el Comité Técnico de la RFEA y dirigida por el responsable de las Categorías Menores, desde donde se hace eco de la preocupación por cómo lograr una mayor participación de jóvenes en el atletismo. Ésta surge en consonancia con la guía IAAF KIDS' ATHLETICS, presentada por la Asociación Internacional de Federaciones de Atletismo (IAAF), que pretende evitar seguir cerrando el programa de prueba para los más jóvenes, como tradicionalmente se ha venido haciendo, a unas distancias, alturas y pesos concretos e inamovibles. Análogamente, se considera que el entorno más adecuado y eficaz para realizar esta promovión es el medio escolar, asociando diversión y competición mediante el juego (García, Durán y Sainz, 2004).

Finalmente, Valero y Conde (2003), plantean una propuesta alternativa a la enseñanza del atletismo en la iniciación deportiva denominada Modelo Ludotécnico, en el que a través de juegos modificads que incluyen una o varias reglas técnicas, los practicantes aprenden las diferentes disciplinas atléticas, evitando la repetición continua del gesto atlético que conduce al aburrimiento y a la pérdida de interés hacia dicha práctica deportiva. El Modelo de Enseñanza Ludotécnico, con una clara voluntad renovadora de la visión del atletismo en el sistema educativo, está pensado tanto para el $3^{\text {er }}$ ciclo de Educación Primaria, como para la etapa de la ESO.

\section{3.- EL ATLETISMO EN EL CURRÍCULUM DE EDUCACIÓN FÍSICA DE LA ESO}

A la hora de analizar el atletismo dentro del currículum de la Educación Física en la enseñanza secundaria obligatoria, se ha centrado especialmente la atención a la normativa existente en la Región de Murcia, ya que cuenta con competencias propias en materia de educación y existen una serie de decretos que así la regulan. En este caso, se busca aportar una fundamentación teórica lo más ajustada al entorno donde se pretende poner en práctica. A tal efecto se presenta la propuesta de secuenciación en la iniciación a la práctica del atletismo a lo largo del sistema educativo, desde la etapa de Primaria 
hasta la Secundaria post-obligatoria, para posteriormente concretar a través de los diferentes elementos curriculares la relación que se puede llegar a establecer con el atletismo.

\section{Secuenciación del deporte del atletismo y sus disciplinas atléticas a lo largo del sistema educativo}

El análisis de la Ley Orgánica 2/2006, de 3 de mayo, de Educación, y de los Reales Decretos y Decretos por los que se establecen las enseñanzas mínimas correspondientes a la Educación Primaria, Secundaria Obligatoria y Post-obligatoria, invitan a que se realice una propuesta de secuenciación de los contenidos del atletismo a lo largo del sistema educativo, centrándose especialmente en la etapa de secundaria obligatoria, con la intención de reflexionar, generar debate y avanzar a este respecto.

El atletismo puede y debe estar presente como parte de los contenidos en el currículum de Primaria, en el segundo y especialmente en el tercer ciclo, dentro del bloque de "Juegos y actividades deportivas", como fracción del contenido "Participación en juegos e iniciación a la práctica de actividades deportivas", contribuyendo al objetivo de "Conocer y valorar la diversidad de actividades físicas, lúdicas y deportivas como elementos culturales, mostrando una actitud crítica tanto desde la perspectiva de participante como de espectador" (Real Decreto 1513/2006). La etapa de la Educación Secundaria Obligatoria, que comprende entre los 12 y 16 años de edad, es el periodo en el que aparece una mayor cantidad de referencias a contenidos de práctica deportiva, distinguiendo entre deportes colectivos, de adversario e individuales. De acuerdo con la taxonomía de los deportes individuales de Batalla y Martínez (2002), se comprueba que el atletismo es el deporte que más posibilidades ofrece (con una gran variedad de disciplinas atléticas) para ponerlo en práctica en los centros educativos, por la disponibilidad de material y espacios para su implementación en las aulas, quedando otras como los deportes náuticos, acuáticos, deportes en la naturaleza y deportes de invierno relegados a su realización en actividades extraescolares.

El objetivo de área que busca "Conocer y practicar actividades y modalidades deportivas individuales, colectivas y de adversario, aplicando los fundamentos reglamentarios, técnicos y tácticos en situaciones de juego, con progresiva autonomía en su ejecución" se encuentra directamente relacionado con los contenidos de "Práctica de juegos regulados y adaptados, convencionales y modificados, populares y tradicionales, que faciliten el aprendizaje de los fundamentos técnicos/tácticos y las estrategias de ataque y defensa comunes a los deportes individuales, de adversario y colectivos", y "Realización de actividades cooperativas y competitivas encaminadas al aprendizaje de los fundamentos técnicos y tácticas de un deporte individual, de adversario y colectivo" para el primer ciclo de secundaria y, en el caso de $3^{\circ}$ y $4^{\circ}$ con los contenidos de "Práctica de actividades encaminadas al aprendizaje de un deporte individual, de adversario o colectivo" y "Realización de actividades encaminadas al aprendizaje y al perfeccionamiento del deporte individual, de adversario o colectivo".

Así, la propuesta que se realiza consiste en la elaboración de una unidad didáctica para cada uno de los cursos de la etapa secundaria, tanto obligatoria como post-obligatoria, donde se presentan las disciplinas que componen el atletismo. Se busca una progresión sencilla, a la vez que segura, donde no se ponga en peligro la integridad del alumnado, atendiendo también a las necesidades de material e instalaciones, planteando en cada sesión una serie de propuestas lúdicas de la disciplina que permita a los niños al final de la misma ejecutar la disciplina decentemente y medir sus propios logros. Puede dedicársele a alguna disciplina más tiempo que el de una única sesión, siempre y cuando suscite suficiente interés y demanda por parte del alumnado, como podría ser el caso del salto de altura o la carrera con obstáculos. Con esta intención, en el tercer ciclo de primaria $\left(5^{\circ}\right.$ y $\left.6^{\circ}\right)$ se apuesta por una unidad didáctica de entre 4 y 6 sesiones comprendiendo las disciplinas de las carreras de velocidad, los relevos, el salto de longitud y el lanzamiento de peso mientras que en el primer ciclo $\left(1^{\circ} \mathrm{y} 2^{\circ}\right.$ de la ESO), esta unidad didáctica podría abarcar entre 9 y 12 sesiones y contendría las disciplinas atléticas para $1^{\circ}$ de la ESO de carreras de velocidad y relevos, salto de longitud y lanzamiento de peso y para $2^{\circ}$ de marcha atlética, carreras con obstáculos y lanzamiento de jabalina. A cada una de ellas se dedicaría entre 3 y 4 sesiones, conocedores de que los aprendizajes técnicos no serán demasiado significativos, pero sí se lograría cumplir con los objetivos didácticos planteados, centrados en conocer y practicar una amplia variedad de disciplinas deportivas. 
En el segundo ciclo ( $3^{\circ}$ y $4^{\circ}$ de la ESO), se persigue ahondar en el conocimiento de disciplinas atléticas que entrañen mayores complejidades técnicas y que exigen unos mayores requerimientos en instalaciones como en el caso del salto de altura y triple salto con una zona de caída bien acolchada, resguardando la integridad de los niños y evitando posibles accidentes, o zonas de terreno amplias como para el caso del lanzamiento de disco y martillo que permitan desarrollar la magnitud de las acciones técnicas, dándole la máxima vistosidad a las grandes distancias que recorren los artefactos sin renunciar a la seguridad de los ejecutantes. A diferencia del ciclo anterior, la propuesta de las disciplinas atléticas a trabajar se presenta más abierta, consciente de que depende en gran medida de las propias posibilidades materiales y de instalaciones del centro, apostando por el salto de altura o el triple salto en $3^{\circ}$ de la ESO y por el lanzamiento de disco o martillo en $4^{\circ}$ de ESO. El número de sesiones destinadas a desarrollar la unidad didáctica que se ha propuesto está comprendido entre 4 y 6 , tiempo suficiente para presentar la disciplina atlética, experimentar una práctica atractiva y divertida, desarrollando los aspectos técnicos y reglamentarios básicos de la misma.

Tabla 1. Secuenciación de las disciplinas atléticas a lo largo del sistema educativo

\begin{tabular}{|c|c|c|c|c|}
\hline ETAPA & $\begin{array}{l}\text { CICLO Y } \\
\text { EDAD }\end{array}$ & CURSO & CONTENIDO & SESIONES \\
\hline \multirow{2}{*}{ PRIMARIA } & \multirow{2}{*}{$\begin{array}{l}\text { Tercer Ciclo } \\
\text { (10-12 años) }\end{array}$} & Quinto & $\begin{array}{l}\text { Velocidad, relevos, } \\
\text { longitud y peso }\end{array}$ & $4-6$ \\
\hline & & Sexto & $\begin{array}{l}\text { Marcha, obstáculos, } \\
\text { altura y jabalina }\end{array}$ & $4-6$ \\
\hline \multirow{4}{*}{$\begin{array}{l}\text { SECUNDARIA } \\
\text { OBLIGATORIA }\end{array}$} & \multirow{2}{*}{$\begin{array}{l}\text { Primer Ciclo } \\
\text { (12-14 años) }\end{array}$} & Primero & $\begin{array}{c}\text { Relevos, longitud y } \\
\text { peso }\end{array}$ & $9-12$ \\
\hline & & Segundo & $\begin{array}{c}\text { Marcha, obstáculos y } \\
\text { jabalina }\end{array}$ & $9-12$ \\
\hline & \multirow{2}{*}{$\begin{array}{l}\text { Segundo Ciclo } \\
\text { (14-16 años) }\end{array}$} & Tercero & Salto de altura/triple & $9-12$ \\
\hline & & Cuarto & $\begin{array}{l}\text { Lanzamiento de } \\
\text { disco/martillo }\end{array}$ & $9-12$ \\
\hline \multirow{2}{*}{$\begin{array}{l}\text { SECUNDARIA } \\
\text { POST- } \\
\text { OBLIGATORIA }\end{array}$} & \multirow{2}{*}{$\begin{array}{l}\text { Ciclo único } \\
\text { (16-18 años) }\end{array}$} & Primero & $\begin{array}{l}\text { Lanzamiento de } \\
\text { martillo }\end{array}$ & $4-6$ \\
\hline & & Segundo & Salto con pértiga & $4-6$ \\
\hline
\end{tabular}

La etapa de la Educación Secundaria Post-obligatoria, es decir, $1^{\circ}$ y $2^{\circ}$ de Bachillerato, se presenta como un periodo educativo, en donde el atletismo tiene cabida dentro del bloque de contenidos denominado "Actividad física, deporte y tiempo libre", en el contenido "Progreso en los fundamentos técnicos y principios tácticos de alguno de los deportes practicados en la etapa anterior" y "Realización de juegos y deportes, con manejo de un implemento", contribuyendo a desarrollar el objetivo de área "Resolver situaciones motrices deportivas, dando prioridad a la toma de decisiones utilizando elementos técnicos aprendidos en la etapa anterior” (Real Decreto 1467/2007). En el caso de que el centro educativo cuente con la materia de Educación Física tanto en $1^{\circ}$ como en $2^{\circ}$ (en este último curso, es una optativa que no es obligatoria ofertar en todos los centros educativos), se propone trabajar en esta etapa las disciplinas más complejas como son el lanzamiento de martillo y el salto con pértiga. Si no existiese la materia en $2^{\circ}$ de Bachillerato, se abordaría en $1^{\circ}$ el salto con pértiga, siempre y cuando sea posible su puesta en práctica, siendo conscientes de que disciplinas como el salto con pértiga, además de ser la más compleja técnicamente, requiere una zona de caída muy específica, y un material (pértigas) para su puesta en práctica costoso y no fácil de conseguir. En cualquier caso, sería la oportunidad para abordar aquellas disciplinas atléticas que no se pudieron ver durante la etapa obligatoria de la ESO, o bien, ahondar en aquellas que el alumnado demanda con mayor insistencia. 
Tabla 2. Ejemplo de unidad didáctica de iniciación al atletismo

\begin{tabular}{|c|c|c|c|c|c|c|}
\hline \multicolumn{7}{|c|}{ UNIDAD DIDÁCTICA: INICIACIÓN AL ATLETISMO } \\
\hline \multirow{4}{*}{$\begin{array}{c}\text { ¿QUÉ } \\
\text { ENSEÑNAR? }\end{array}$} & \multicolumn{2}{|c|}{ Objetivos Didácticos } & \multicolumn{4}{|c|}{$\begin{array}{l}\text { - Iniciarse en el aprendizaje de los fundamentos técnicos de una serie } \\
\text { de disciplinas atléticas. } \\
\text { - Conocer las técnicas y normas básicas del atletismo. } \\
\text { - Practicar una serie de disciplinas atléticas a partir de las formas } \\
\text { jugadas. } \\
\text { - Establecer relaciones constructivas y equilibradas con los demás. } \\
\text { - Contribuir a la mejora de las habilidades motrices y de la condición } \\
\text { física. }\end{array}$} \\
\hline & \multirow{3}{*}{ Contenidos } & Saber & \multicolumn{4}{|c|}{$\begin{array}{l}\text { - Conocimiento de la técnica básica de las carreras de relevos, del } \\
\text { salto de longitud y lanzamiento de peso. } \\
\text { - Conocimiento del reglamento básico de las disciplinas atléticas } \\
\text { abordadas. }\end{array}$} \\
\hline & & $\begin{array}{c}\text { Saber ser y } \\
\text { estar }\end{array}$ & \multicolumn{4}{|c|}{$\begin{array}{l}\text { - Interés por la práctica atlética aceptando los diferentes niveles de } \\
\text { destreza, tanto en sí mismo como en los otros. } \\
\text { - Respeto a los compañeros y al material. } \\
\text { - Aceptación de las normas y reglas del atletismo y del papel que } \\
\text { corresponda desempeñar como jugador. }\end{array}$} \\
\hline & & Saber hacer & \multicolumn{4}{|c|}{$\begin{array}{l}\text { - Práctica adaptada de las carreras de relevos, del salto de longitud y } \\
\text { del lanzamiento de peso. } \\
\text { - Utilización de reglas para la organización de situaciones colectivas } \\
\text { de juego y de estrategias básicas. } \\
\text { - Aumento de la precisión y economía de las habilidades. }\end{array}$} \\
\hline \multirow{3}{*}{$\begin{array}{l}\text { ¿CÓMO Y } \\
\text { CUÁNDO } \\
\text { ENSEÑAR? }\end{array}$} & \multirow{3}{*}{ Metodología } & Actividades & \multicolumn{4}{|c|}{$\begin{array}{l}\text { - Sesiones } 1^{\mathrm{a}} \text { hasta } 4^{\mathrm{a}} \text { : Propuestas lúdicas de actividades relacionadas } \\
\text { con las carreras de relevos. } \\
\text { - Sesiones } 5^{\mathrm{a}} \text { hasta } 8^{\mathrm{a}} \text { : Propuestas lúdicas de actividades relacionadas } \\
\text { con el salto de longitud. } \\
\text { - Sesiones } 9^{\mathrm{a}} \text { hasta } 12^{\mathrm{a}} \text { : Propuestas lúdicas de actividades } \\
\text { relacionadas con el lanzamiento de peso. }\end{array}$} \\
\hline & & $\begin{array}{l}\text { Material y } \\
\text { recursos }\end{array}$ & \multicolumn{4}{|c|}{$\begin{array}{l}\text { Pelotas de diferentes tamaños, picas, colchonetas quitamiedos, } \\
\text { simuladores de peso, vallas, conos, bancos suecos, globos, pelotas de } \\
\text { espuma, elásticos, cuerdas, pandero, aros de diferentes tamaños, } \\
\text { testigos, balones medicinales, saltómetros, trampolín, cinta adhesiva } \\
\text { y todo tipo de material alternativo. }\end{array}$} \\
\hline & & Estrategias & \multicolumn{4}{|c|}{$\begin{array}{l}\text { Modelo ludotécnico basado en un esquema de sesión compuesto por } \\
\text { cuatro partes: } 1^{\mathrm{a}} \text { presentación global y propuesta de desafíos, } 2^{\mathrm{a}} \\
\text { propuestas ludotécnicas, } 3^{\mathrm{a}} \text { propuestas globales y } 4^{\mathrm{a}} \text { reflexión y } \\
\text { puesta en común. }\end{array}$} \\
\hline \multirow[t]{2}{*}{$\begin{array}{l}\text { ¿QUÉ, } \\
\text { CÓMO Y } \\
\text { CUÁNDO } \\
\text { EVALUAR? }\end{array}$} & \multirow[t]{2}{*}{$\begin{array}{c}\text { Evaluación } \\
\text { del } \\
\text { aprendizaje }\end{array}$} & Criterios & $\begin{array}{l}\text { - Si practica las } \\
\text { diferentes disciplinas } \\
\text { del atletismo. } \\
\text { - Si conoce la técnica y } \\
\text { normas básicas del } \\
\text { atletismo. } \\
\text { - Si establecen } \\
\text { relaciones } \\
\text { constructivas con los } \\
\text { demás. }\end{array}$ & \multirow[t]{2}{*}{$\begin{array}{l}\text { Evaluación } \\
\text { de la } \\
\text { enseñanza }\end{array}$} & Criterios & $\begin{array}{l}\text { Si los objetivos } \\
\text { han sido } \\
\text { adecuados al } \\
\text { nivel del grupo y } \\
\text { las actividades } \\
\text { motivantes. }\end{array}$ \\
\hline & & Instrumentos & $\begin{array}{l}\text { - Observación } \\
\text { sistemática. } \\
\text { - Corrección de } \\
\text { actividades. } \\
\text { - Trabajo en grupos. } \\
\text { - Propuestas lúdicas. }\end{array}$ & & Instrumentos & $\begin{array}{l}\text { Cuestionario } \\
\text { acerca del interés } \\
\text { por la práctica de } \\
\text { atletismo. }\end{array}$ \\
\hline
\end{tabular}


A modo de cuadro resumen, se trata de sintetizar las ideas plasmadas en los párrafos anteriores y que se plantea como propuesta de secuenciación de los contenidos de Atletismo a lo largo del sistema educativo (Tabla 1 ).

\section{4.- RELACIÓN DEL ATLETISMO CON LAS ENSEÑANZAS MÍNIMAS DE LA EDUCACIÓN SECUNDARIA OBLIGATORIA}

Según el Real Decreto 1631/2006, de 29 de diciembre, por el que se establecen las enseñanzas mínimas correspondientes a la Educación Secundaria Obligatoria, las enseñanzas mínimas son los aspectos básicos del currículo referidos a los objetivos, las competencias básicas, los contenidos y los criterios de evaluación.

Continuando con el ejercicio de reflexión, análisis y síntesis planteado, resulta oportuno relacionar el atletismo con los elementos básicos del currículum, si bien, en un intento de ser lo más pragmáticos posible y buscando la máxima aplicabilidad de las aportaciones que aquí se hacen a la realidad actual, dentro de este apartado se va a ir poniendo en conexión los elementos del currículum con una unidad didáctica hipotética que se aplicaría en $1^{\circ}$ de la ESO para la iniciación a las carreras de relevos, al salto de longitud y al lanzamiento de peso (Tabla 2).

\section{Relación del atletismo con los objetivos de etapa y materia}

Los objetivos de etapa son enunciados o intenciones educativas que fijan las capacidades que el alumnado ha de haber adquirido basándose en las diferentes áreas al final de la etapa correspondiente, mientras que los objetivos de materia se refieren a una determinada área concreta (Díaz Lucea, 1995); la Educación Física en el caso que nos atañe. Para la Región de Murcia, éstos se recogen en el Decreto 291/2007, de 14 de septiembre, por el que se establece el currículo de la Educación Secundaria obligatoria en la Comunidad Autónoma de la Región de Murcia.

Realizando un análisis similar al empleado en párrafos anteriores, se ubican en la siguiente Tabla 3 los objetivos de la materia y se relacionan con los de la unidad didáctica, pudiéndose observar cómo éstos últimos contribuye a alcanzar los de la materia. Asimismo, también se puede establecer la conexión con los propios objetivos de etapa.

\section{Relación del atletismo con las competencias básicas}

Conforme al Real Decreto 1631/2006, se entiende por competencias básicas del currículo aquellas que debe haber desarrollado un joven al finalizar la enseñanza obligatoria para poder lograr su realización personal, ejercer la ciudadanía activa, incorporarse a la vida adulta de manera satisfactoria y ser capaz de desarrollar un aprendizaje permanente a lo largo de la vida. Las competencias básicas permiten poner el acento en aquellos aprendizajes que se consideran imprescindibles, desde un planteamiento integrador y orientado a la aplicación de los saberes adquiridos.

Su inclusión tiene varias finalidades: en primer lugar, integrar los diferentes aprendizajes, tanto los formales, incorporados a las diferentes áreas o materias, como los informales y no formales; en segundo lugar, permite a todos los estudiantes integrar sus aprendizajes, ponerlos en relación con distintos tipos de contenidos y utilizarlos de manera efectiva cuando les resulten necesarios en diferentes situaciones y contextos; y, por último, orientar la enseñanza, al permitir identificar los contenidos y los criterios de evaluación que tienen carácter imprescindible y, en general, inspirar las distintas decisiones relativas al proceso de enseñanza y de aprendizaje. En este sentido se distinguen 8 competencias básicas: a) competencia en comunicación lingüística, b) matemática, c) conocimiento e interacción con el mundo físico, d) tratamiento de la información y competencia digital, e) social y ciudadana, f) cultural y artística, g) para aprender a aprender y h) autonomía e iniciativa personal.

Desde la unidad didáctica propuesta se plantean situaciones especialmente favorables a la adquisición de la competencia social y ciudadana, facilitando la integración y fomentando el respeto, a la vez que contribuye al desarrollo de la cooperación, la igualdad y el trabajo en equipo. El atletismo contribuye a la integración en un proyecto común y aceptación de las diferencias y limitaciones de los participantes, siguiendo normas democráticas en la organización del grupo y asumiendo cada integrante sus propias responsabilidades. El cumplimiento de las normas y de los reglamentos que 
rigen las disciplinas atléticas colabora en la aceptación de los códigos de conducta propios de una sociedad.

La competencia en el conocimiento y la interacción con el mundo físico, partiendo del conocimiento del cuerpo humano, de la naturaleza y de la interacción de los hombres y mujeres con ella, permite argumentar racionalmente las consecuencias de unos y otros modos de vida y adoptar una disposición a una vida física y mental saludable en un entorno natural y social también saludable. Asimismo, supone considerar la doble dimensión (individual y colectiva) de la salud y mostrar actitudes de responsabilidad y respeto hacia los demás y hacia uno mismo. El atletismo proporciona conocimientos y destrezas sobre determinados hábitos saludables que acompañarán a los jóvenes más allá de la etapa obligatoria. Aporta criterios para el mantenimiento y mejora de la condición física, sobre todo de aquellas cualidades físicas asociadas a la salud: resistencia cardiovascular, fuerzaresistencia y flexibilidad. La unidad didáctica de atletismo ayuda de forma destacable a la consecución de la autonomía e iniciativa personal, fundamentalmente en la medida en que el alumnado se enfrenta a situaciones en las que debe manifestar autosuperación, perseverancia y actitud positiva ante tareas de cierta dificultad técnica o en la mejora del propio nivel de condición física, responsabilidad y honestidad en la aplicación de las reglas y capacidad de aceptación de los diferentes niveles de condición física y de ejecución motriz dentro del grupo.

Tabla 3. Relación entre objetivos didácticos, de materia y de etapa.

\begin{tabular}{|c|c|c|}
\hline $\begin{array}{c}\text { OBJ. DI- } \\
\text { DÁCTICOS }\end{array}$ & OBJ. DE MATERIA & OBJ. DE ETAPA \\
\hline $\begin{array}{l}\text { Iniciarse en el } \\
\text { aprendizaje de } \\
\text { los fundamentos } \\
\text { técnicos de una } \\
\text { serie de } \\
\text { disciplinas } \\
\text { atléticas. }\end{array}$ & $\begin{array}{l}\text { a) Participar y colaborar de manera activa, con } \\
\text { regularidad y eficiencia, en las actividades } \\
\text { programadas, con independencia desnivel de } \\
\text { habilidad y capacidad personal y valorando los } \\
\text { aspectos de relación que fomentan; mostrando } \\
\text { una actitud de respeto y tolerancia hacia todos } \\
\text { los miembros de la comunidad educativa. b) } \\
\text { Aumentar las propias posibilidades de } \\
\text { rendimiento motor mediante la mejora de las } \\
\text { capacidades, tanto físicas como motrices, } \\
\text { desarrollando actitudes de autoexigencia y } \\
\text { superación personal. } \\
\text { Conocer y practicar actividades y modalidades } \\
\text { deportivas individuales, colectivas y de } \\
\text { adversario, aplicando los fundamentos } \\
\text { reglamentarios, técnicos y tácticos en situaciones } \\
\text { de juego, con progresiva autonomía en su } \\
\text { ejecución. }\end{array}$ & $\begin{array}{l}\text { Conocer el funcionamiento del cuerpo } \\
\text { humano, respetar las diferencias, así } \\
\text { como valorar los efectos beneficiosos } \\
\text { para la salud del ejercicio físico y la } \\
\text { adecuada alimentación, incorporando } \\
\text { la práctica del deporte para favorecer } \\
\text { el desarrollo personal y social. }\end{array}$ \\
\hline $\begin{array}{l}\text { Conocer las } \\
\text { técnicas y } \\
\text { normas básicas } \\
\text { de las diferentes } \\
\text { disciplinas } \\
\text { atléticas. }\end{array}$ & $\begin{array}{l}\text { a) Conocer y practicar actividades y } \\
\text { modalidades deportivas individuales, colectivas } \\
\text { y de adversario, aplicando los fundamentos } \\
\text { reglamentarios, técnicos y tácticos en situaciones } \\
\text { de juego, con progresiva autonomía en su } \\
\text { ejecución. }\end{array}$ & $\begin{array}{l}\text { Conocer, asumir responsablemente y } \\
\text { ejercer sus derechos y deberes en el } \\
\text { respeto a los demás, practicar la } \\
\text { tolerancia, la cooperación y } \\
\text { solidaridad entre las personas y los } \\
\text { grupos, ejercitarse en el diálogo } \\
\text { afianzando los derechos humanos } \\
\text { como valores comunes de una } \\
\text { sociedad plural, abierta y democrática. }\end{array}$ \\
\hline
\end{tabular}




\begin{tabular}{|c|c|c|}
\hline $\begin{array}{l}\text { Practicar una } \\
\text { serie de } \\
\text { disciplinas } \\
\text { atléticas a partir } \\
\text { de las formas } \\
\text { jugadas. }\end{array}$ & $\begin{array}{l}\text { a) Participar y colaborar de manera activa, con } \\
\text { regularidad y eficiencia, en las actividades } \\
\text { programadas, con independencia del nivel de } \\
\text { habilidad y capacidad personal y valorando los } \\
\text { aspectos de relación que fomentan; mostrando } \\
\text { una actitud de respeto y tolerancia hacia todos } \\
\text { los miembros de la comunidad educativa. b) } \\
\text { Aumentar las propias posibilidades de } \\
\text { rendimiento motor mediante la mejora de las } \\
\text { capacidades, tanto físicas como motrices, } \\
\text { desarrollando actitudes de autoexigencia y } \\
\text { superación personal. } \\
\text { Conocer y practicar actividades y modalidades } \\
\text { deportivas individuales, colectivas y de } \\
\text { adversario, aplicando los fundamentos } \\
\text { reglamentarios, técnicos y tácticos en situaciones } \\
\text { de juego, con progresiva autonomía en su } \\
\text { ejecución. }\end{array}$ & $\begin{array}{l}\text { Conocer el funcionamiento del cuerpo } \\
\text { humano, respetar las diferencias, así } \\
\text { como valorar los efectos beneficiosos } \\
\text { para la salud del ejercicio físico y la } \\
\text { adecuada alimentación, incorporando } \\
\text { la práctica del deporte para favorecer } \\
\text { el desarrollo personal y social. }\end{array}$ \\
\hline $\begin{array}{l}\text { Establecer } \\
\text { relaciones } \\
\text { constructivas y } \\
\text { equilibradas con } \\
\text { los demás. }\end{array}$ & $\begin{array}{l}\text { a) Participar y colaborar de manera activa, con } \\
\text { regularidad y eficiencia, en las actividades } \\
\text { programadas, con independencia desnivel de } \\
\text { habilidad y capacidad personal y valorando los } \\
\text { aspectos de relación que fomentan; mostrando } \\
\text { una actitud de respeto y tolerancia hacia todos } \\
\text { los miembros de la comunidad educativa. b) } \\
\text { Mostrar habilidades y actitudes sociales de } \\
\text { respeto, trabajo en equipo y deportividad en la } \\
\text { participación de actividades, juegos y deportes, } \\
\text { independientemente de las diferencias culturales, } \\
\text { sociales y de habilidad. }\end{array}$ & $\begin{array}{l}\text { a) Conocer, asumir responsablemente } \\
\text { y ejercer sus derechos y deberes en el } \\
\text { respeto a los demás, practicar la } \\
\text { tolerancia, la cooperación y } \\
\text { solidaridad entre las personas y los } \\
\text { grupos, ejercitarse en el diálogo } \\
\text { afianzando los derechos humanos } \\
\text { como valores comunes de una } \\
\text { sociedad plural, abierta y democrática. } \\
\text { b) Fortalecer sus capacidades afectivas } \\
\text { en todos los ámbitos de la } \\
\text { personalidad, así como fomentar } \\
\text { actitudes que favorezcan la } \\
\text { convivencia y eviten la violencia en } \\
\text { los ámbitos escolar, familiar social, } \\
\text { resolviendo pacíficamente los } \\
\text { conflictos. }\end{array}$ \\
\hline $\begin{array}{l}\text { Contribuir a la } \\
\text { mejora de las } \\
\text { habilidades } \\
\text { motrices y de la } \\
\text { condición física. }\end{array}$ & $\begin{array}{l}\text { a) Aumentar las propias posibilidades de } \\
\text { rendimiento motor mediante la mejora de las } \\
\text { capacidades, tanto físicas como motrices, } \\
\text { desarrollando actitudes de autoexigencia y } \\
\text { superación personal. } \\
\text { Conocer el cuerpo y sus necesidades, adoptando } \\
\text { una actitud favorable y consecuente frente a las } \\
\text { actividades dirigidas a la mejora de la condición } \\
\text { física, la salud y la calidad de vida, haciendo un } \\
\text { tratamiento diferenciado de cada capacidad. }\end{array}$ & $\begin{array}{l}\text { a) Adquirir, desarrollar y consolidar } \\
\text { hábitos de disciplina, estudio y trabajo } \\
\text { individual y en equipo como condición } \\
\text { necesaria para una realización eficaz } \\
\text { de las tareas del aprendizaje y como } \\
\text { medio de desarrollo personal. } \\
\text { b) Desarrollar el espíritu emprendedor } \\
\text { y la confianza en sí mismo, la } \\
\text { participación, el sentido crítico, la } \\
\text { iniciativa personal y la capacidad para } \\
\text { aprender a aprender, para planificar, } \\
\text { para tomar decisiones y para asumir } \\
\text { responsabilidades, valorando el } \\
\text { esfuerzo con la finalidad de superar las } \\
\text { dificultades. } \\
\text { funcionamiento del cuerpo humano, } \\
\text { respetar las diferencias, así como } \\
\text { valorar los efectos beneficiosos para la } \\
\text { salud del ejercicio físico y la adecuada } \\
\text { alimentación, incorporando la práctica } \\
\text { del deporte para favorecer el desarrollo } \\
\text { personal y social. }\end{array}$ \\
\hline
\end{tabular}


Además, también contribuye a la adquisición de la competencia cultural y artística, mediante el reconocimiento y la valoración de las manifestaciones culturales de la motricidad humana, tal como el deporte del atletismo y su consideración como parte del patrimonio cultural de los pueblos. La unidad didáctica está comprometida con la adquisición de una actitud abierta y respetuosa ante el fenómeno del atletismo como espectáculo, mediante el análisis y la reflexión crítica ante la discriminación por razones de sexo, raza y religión u otras contrarias a la dignidad humana que en él se producen. La competencia para aprender a aprender tiene dos dimensiones. Por un lado, la adquisición de la conciencia de las propias capacidades (intelectuales, emocionales, físicas), del proceso y las estrategias necesarias para desarrollarlas, así como de lo que se puede hacer por uno mismo y de lo que se puede hacer con ayuda de otras personas o recursos. Por otro lado, disponer de un sentimiento de competencia personal, que redunda en la motivación, la confianza en uno mismo y el gusto por aprender. La unidad didáctica de iniciación al atletismo ayuda a desarrollar habilidades para el trabajo en equipo en las diferentes tareas y contribuye a adquirir aprendizajes técnicos y estratégicos que son generalizables para varias actividades deportivas. Este vínculo entre los objetivos didácticos que persiguen la unidad didáctica propuesta a través del atletismo y las competencias básicas queda recogido en la Tabla 4.

Tabla 4. Relación entre objetivos didácticos y competencias básicas.

\begin{tabular}{|l|l|}
\hline \multicolumn{1}{|c|}{ OBJETIVOS DIDÁCTICOS } & \multicolumn{1}{c|}{ COMPETENCIAS BÁSICAS } \\
\hline $\begin{array}{l}\text { Iniciarse en el aprendizaje de los } \\
\text { fundamentos técnicos de una serie de } \\
\text { disciplinas atléticas. }\end{array}$ & $\begin{array}{l}\text { Autonomía e iniciativa personal } \\
\text { Competencia para aprender a aprender }\end{array}$ \\
\hline $\begin{array}{l}\text { Conocer las técnicas y normas básicas de } \\
\text { las diferentes disciplinas atléticas. }\end{array}$ & $\begin{array}{l}\text { Competencia cultural y artística } \\
\text { Competencia para aprender a aprender }\end{array}$ \\
\hline $\begin{array}{l}\text { Practicar una serie de disciplinas } \\
\text { atléticas a partir de las formas jugadas. }\end{array}$ & $\begin{array}{l}\text { Conocimiento y la interacción con el mundo físico } \\
\text { Competencia para aprender a aprender }\end{array}$ \\
\hline $\begin{array}{l}\text { Establecer relaciones constructivas y } \\
\text { equilibradas con los demás. }\end{array}$ & $\begin{array}{l}\text { Competencia social y ciudadana } \\
\text { Conocimiento y la interacción con el mundo físico } \\
\text { Autonomía e iniciativa personal } \\
\text { Competencia para aprender a aprender }\end{array}$ \\
\hline $\begin{array}{l}\text { Contribuir a la mejora de las habilidades } \\
\text { motrices y de la condición física. }\end{array}$ & $\begin{array}{l}\text { Conocimiento y la interacción con el mundo físico } \\
\text { Competencia para aprender a aprender }\end{array}$ \\
\hline
\end{tabular}

\section{Relación del atletismo con los contenidos}

Tradicionalmente los contenidos se han presentado cargados de significación intelectualista, reflejados bajo formas de materias o asignaturas, mientras que en la actualidad se tiende a una acepción mucho más amplia que abarca las finalidades de la escolaridad (Contreras, 1998). Los contenidos han de tener una concepción más abierta, superando la noción de contenido basado sólo en modelos conceptuales y ampliándose a otros campos. El atletismo debe estar al servicio del profesorado para conseguir las metas que persigue la educación a través del saber, saber ser y estar y saber hacer.

El contenido del atletismo está enmarcado dentro del bloque de contenidos denominado “Juegos y deportes. Cualidades motrices básicas”, con referencias directas a la práctica y desarrollo de 
algún deporte individual. Entrando más en detalle y siguiendo el Decreto 291/2007, los contenidos para cada uno de los cursos son los siguientes:

$1^{\circ}$ y $2^{\circ}$ curso:

- Práctica de juegos regulados y adaptados, convencionales y modificados, populares y tradicionales, que faciliten el aprendizaje de los fundamentos técnicos/tácticos y las estrategias de ataque y defensa comunes a los deportes individuales, de adversario y colectivos.

- Realización de actividades cooperativas y competitivas encaminadas al aprendizaje de los fundamentos técnicos y tácticas de un deporte individual, de adversario y colectivo.

$3^{\circ}$ curso:

- Aspectos técnicos, tácticos y reglamentarios de los deportes que tienen que practicarse.

- Práctica de actividades encaminadas al aprendizaje de un deporte individual, de adversario o colectivo.

$4^{\circ}$ curso:

- Realización de actividades encaminadas al aprendizaje y al perfeccionamiento del deporte individual, de adversario o colectivo.

De manera transversal, el atletismo desarrolla otros contenidos ubicados en el bloque de "Juegos y deportes. Cualidades motrices personales", como pueden ser las habilidades motrices y en el bloque de "Condición física y salud”, la práctica de resistencia aeróbica, de fuerza general, flexibilidad y velocidad.

\section{Relación del atletismo con los criterios de evaluación}

Desde el Real Decreto 1631/2006, se manifiesta la importancia de los criterios de evaluación en el actual sistema educativo, pues además de permitir la valoración del tipo y grado de aprendizaje adquirido, se convierten en referente fundamental para valorar la adquisición de las competencias básicas, indicando que dicha evaluación será continua y diferenciada según las distintas materias del currículo.

Según Contreras (1997), la evaluación supone un proceso por el cual se obtiene información que se utiliza para tomar decisiones o efectuar juicios, tanto sobre la enseñanza-aprendizaje, como sobre la estrategia docente empleada. Por tanto, la evaluación se ha de entender como una actividad básicamente valorativa e investigadora, facilitadora del cambio educativo y del desarrollo profesional del docente, clave para regular el proceso de adaptación y contextualización del currículum en cada comunidad educativa, donde los principios que han de orientarla son:

1. Carácter procesual y continuo.

2. Adecuación a las características de la comunidad escolar y a las de los participantes.

3. Concienciación de la totalidad de elementos que entran a formar parte del hecho educativo.

4. Consideración de la singularidad de cada individuo.

5. Cualitativa y explicativa, respetando la intimidad de los participantes en el proceso evaluador.

Dentro de cada materia, se establecen unos criterios de evaluación por curso, siendo los más cercanos al atletismo, "Mejorar la ejecución de los aspectos técnicos fundamentales de un deporte individual, aceptando el nivel alcanzado" en $1^{\circ}$ y, "Participar en la organización y puesta en práctica de torneos en los que se practicarán deportes y actividades físicas realizadas a lo largo de la etapa” en $4^{\circ}$. Con la intención de relacionar las competencias básicas con los criterios e instrumentos de evaluación de la unidad didáctica, se ha elaborado una tabla (tabla 5), que trata de ponerlos en conexión. 
Tabla 5. Relación entre competencias básicas, criterios e instrumentos de evaluación de la unidad didáctica de atletismo.

\begin{tabular}{|c|c|c|}
\hline COMPETENCIAS BÁSICAS & CRITERIOS DE EVALUACIÓN & $\begin{array}{l}\text { INSTRUMENTOS DE } \\
\text { EVALUACIÓN }\end{array}$ \\
\hline \multirow{2}{*}{$\begin{array}{l}\text { Conocimiento y la interacción } \\
\text { con el mundo físico }\end{array}$} & $\begin{array}{c}\text { Practica las diferentes disciplinas } \\
\text { del atletismo }\end{array}$ & Observación sistemática \\
\hline & $\begin{array}{l}\text { Establece relaciones constructivas } \\
\text { con los demás }\end{array}$ & $\begin{array}{c}\text { Observación sistemática } \\
\text { Trabajo en grupos: propuestas } \\
\text { lúdicas }\end{array}$ \\
\hline \multirow{2}{*}{$\begin{array}{l}\text { Competencia social y } \\
\text { ciudadana }\end{array}$} & $\begin{array}{c}\text { Conoce la técnica y normas básicas } \\
\text { de las disciplinas atléticas }\end{array}$ & $\begin{array}{l}\text { Observación sistemática } \\
\text { Corrección de actividades }\end{array}$ \\
\hline & $\begin{array}{l}\text { Establece relaciones constructivas } \\
\text { con los demás }\end{array}$ & $\begin{array}{c}\text { Observación sistemática } \\
\text { Trabajo en grupos: propuestas } \\
\text { lúdicas } \\
\end{array}$ \\
\hline $\begin{array}{c}\text { Autonomía e iniciativa } \\
\text { personal }\end{array}$ & $\begin{array}{l}\text { Establece relaciones constructivas } \\
\text { con los demás }\end{array}$ & $\begin{array}{c}\text { Observación sistemática } \\
\text { Trabajo en grupos: propuestas } \\
\text { lúdicas }\end{array}$ \\
\hline $\begin{array}{c}\text { Competencia cultural y } \\
\text { artística }\end{array}$ & $\begin{array}{c}\text { Conoce la técnica y normas básicas } \\
\text { de las disciplinas atléticas }\end{array}$ & $\begin{array}{c}\text { Observación sistemática } \\
\text { Corrección de actividades }\end{array}$ \\
\hline \multirow{2}{*}{$\begin{array}{c}\text { Competencia para aprender a } \\
\text { aprender }\end{array}$} & $\begin{array}{c}\text { Practica las diferentes disciplinas } \\
\text { del atletismo }\end{array}$ & Observación sistemática \\
\hline & $\begin{array}{c}\text { Conoce la técnica y normas básicas } \\
\text { de las disciplinas atléticas }\end{array}$ & $\begin{array}{l}\text { Observación sistemática } \\
\text { Corrección de actividades }\end{array}$ \\
\hline
\end{tabular}

\section{5.- CONCLUSIONES}

Este trabajo resalta el valor educativo que posee el deporte del atletismo, entendido como un instrumento al servicio del docente que contribuye a lograr una formación integral del alumnado. El atletismo como contenido curricular de la materia de Educación Física, adquiere una especial relevancia al final de la etapa de la Educación Primaria y a lo largo de la Educación Secundaria, formando parte de los contenidos del currículum.

En este documento se plantea una propuesta didáctica fundamentada especialmente en la actual Ley Orgánica sobre Educación, Reales Decretos y Decretos vigentes, estableciéndose una progresión de los contenidos abarcando varias etapas educativas, en la que se relacionan los objetivos de etapa, con los de la materia de educación física y con los didácticos de los contenidos relacionados con el atletismo, así como con las competencias básicas, criterios e instrumentos de evaluación.

Finalmente, merece especial mención el ejemplo de unidad didáctica de iniciación al atletismo que se incluye en este trabajo, cuyo principal objetivo es servir de borrador para aquellos docentes que afines al planteamiento realizado en este texto, deseen poner en práctica en su centro educativo alguna de las disciplinas del atletismo, sin olvidar en ningún momento, que habrán de hacer las pertinentes adaptaciones al material e instalaciones, así como a las características particulares de sus discentes.

\section{6.- REFERENCIAS BIBLIOGRÁFICAS}

Álvarez, C. (1994). Atletismo Básico. Una orientación pedagógica. Madrid: Gymnos.

Batalla, A. \& Martínez, P. (2002). Deportes individuales. Barcelona: Inde.

Calzada, A. (1999). Manuales para la enseñanza. Iniciación al atletismo. Madrid: Gymnos.

Contreras, O.R. (1997). Didáctica de la Educación Física. En Castejón, F.J. Manual del Maestro Especialista en Educación Física. Madrid: Pila Teleña.

Contreras, O.R. (1998). Didáctica de la Educación Física. Un enfoque constructivista. Barcelona: Inde.

Curtner-Smith, M. \& Sofo, S. (2004). Preservice teachers' conceptions of teaching within sport education and multi-activity units. Sport, Education and Society, 9 (3), 347-377. 
Decreto 291/2007, de 14 de septiembre, por el que se establece el currículo de la Educación Secundaria obligatoria en la Comunidad Autónoma de la Región de Murcia. (BORM n 221, 24 de septiembre de 2007).

Díaz Lucea, J. (1995). El currículum de la Educación Física en la Reforma Educativa. Barcelona: Inde.

García, J., Durán J.P. \& Sainz A. (2004). Jugando al atletismo. Madrid: Real Federación Española de Atletismo.

Hastie, P.A., Martínez de Ojeda, D. \& Calderón, A. (2011). A review of research on Sport Education: 2004 to the present. Physical Education \& Sport Pedagogy, 16(2), 103-132.

Hegedus, J. (1988). Técnicas atléticas. Buenos Aires: Stadium.

Hubiche, J.L. \& Pradet, M. (1999). Comprender el atletismo. Su práctica y su enseñanza. Barcelona: Paidotribo.

Kinchin, G. D. (2006). Sport education: A review of the research. En D. Kirk, M., O’Sullivan y D. Macdonald (Eds.), Handbook of research in physical education (pp. 596-609). Thousand Oaks, CA: Sage.

Ley Orgánica 2/2006, de 3 de mayo, de Educación. (BOE, nº 106, 4 de mayo de 2006).

Méndez, A. (2009). Modelos actuales de iniciación deportiva. Unidades didácticas sobre deportes de invasión. Sevilla: Wanceulen.

Murer, K. (1992). 1000 ejercicios y juegos de atletismo. Barcelona: Hispano Europea.

Polischuk, V. (1996). Atletismo. Iniciación y perfeccionamiento. Barcelona: Paidotribo.

Real Decreto 1467/2007, de 2 de noviembre, por el que se establece la estructura del bachillerato y se fijan sus enseñanzas mínimas. (BOE, $n^{\circ}$ 266, 6 de noviembre de 2007).

Real Decreto 1513/2006, de 7 de diciembre, por el que se establecen las enseñanzas mínimas de la Educación primaria. (BOE ${ }^{\circ} 293,8$ de diciembre de 2006).

Real Decreto 1631/2006, de 29 de diciembre, por el que se establecen las enseñanzas mínimas correspondientes a la Educación Secundaria Obligatoria. (BOE n ${ }^{\circ}$ 5, 5 de enero de 2007).

Rius, J. (1989). Metodología del Atletismo. Barcelona: Paidotribo.

Schmolinsky, G. (1981). Atletismo. Madrid: Pila Teleña.

Siedentop, D. (1994). Sport education: Quality P.E. through positive sport experiences. Champaign, IL: Human Kinetics.

Siedentop, D., Hastie, P.A. \& Van der Mars, H. (2011). Complete guide to Sport Education (2nd ed.). Champaign, IL: Human Kinetics.

Valero, A. (2004). Situación actual de la metodología en la iniciación deportiva al atletismo. Revista de Educación Física. Renovar la teoría y la práctica, 94, 13-20.

Valero, A. \& Conde, J.L. (2003). La iniciación atletismo a través de los juegos. Málaga: Aljibe.

Vinuesa, M. \& Coll, J. (1997). Tratado de Atletismo. Madrid: Esteban Sanz. 